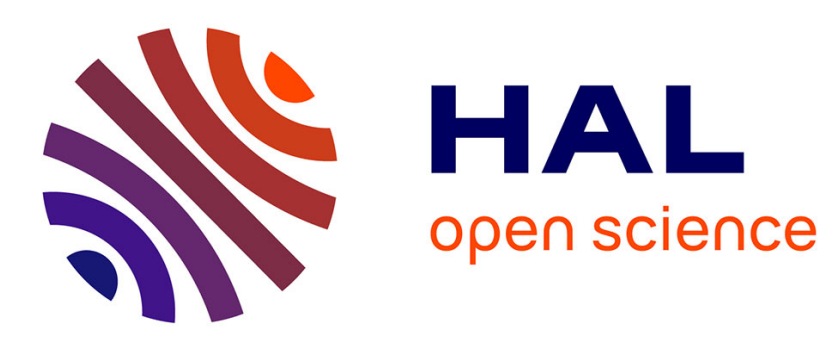

\title{
Measuring hydrogen with fast neutrons: Application to organic materials identification in cargo containers
}

\author{
C. Carasco, B. Perot, A. Sardet
}

\section{To cite this version:}

C. Carasco, B. Perot, A. Sardet. Measuring hydrogen with fast neutrons: Application to organic materials identification in cargo containers. Nuclear Instruments and Methods in Physics Research Section A: Accelerators, Spectrometers, Detectors and Associated Equipment, 2020, 951, pp.163030. 10.1016/j.nima.2019.163030 . cea-02562320

HAL Id: cea-02562320

https://hal-cea.archives-ouvertes.fr/cea-02562320

Submitted on 4 May 2020

HAL is a multi-disciplinary open access archive for the deposit and dissemination of scientific research documents, whether they are published or not. The documents may come from teaching and research institutions in France or abroad, or from public or private research centers.
L'archive ouverte pluridisciplinaire $\mathbf{H A L}$, est destinée au dépôt et à la diffusion de documents scientifiques de niveau recherche, publiés ou non, émanant des établissements d'enseignement et de recherche français ou étrangers, des laboratoires publics ou privés. 


\title{
Measuring hydrogen with fast neutrons: Application to organic materials identification in cargo containers
}

\author{
C. Carasco, B. Pérot, A. Sardet
}

Significance ${ }_{(120 \text { mots max, doit êtrel lisible par des estudiants qui ne sont pas dans } 1 \text { e domaine) }}$

We present a demonstration by simulation of a non-destructive neutron interrogation system capable of identifying organic compounds in cargo containers. Unlike previous systems, which tried identifying organics molecules using only carbon, nitrogen and oxygen neutron-induced gamma rays, our approach allows determining the fraction of hydrogen although no hydrogen specific gamma ray can be measured. Since hydrogen manifests in the gamma ray spectrum in a complex way through changes in the amplitude of carbon, oxygen and nitrogen gamma ray peaks, we discovered that a neural network can cope with the problem complexity and allow interpreting the peaks amplitudes in term of hydrogen fraction. Such a system can improve the reliability of cargo containers screening by reducing false alarms.

\section{Abstract ${ }_{250}$ mots max, comprénensible par tout scientififue)}

To inspect cargo containers, $\mathrm{X}$-ray imaging can be complemented by fast neutron interrogation to provide indication concerning the nature of the transported goods. Organic goods are of special interest since they constitute a significant part of the merchandises. In addition, in the context of NRBC-E threats search in cargo containers, a nondestructive inspection system should also be able to detect explosives. Until now, fast neutron interrogation systems identify organic materials using characteristic neutron-induced gamma-ray peaks of carbon, oxygen and nitrogen. But identifying organics in this way can lead to ambiguities since no hydrogen peak can be measured with fast neutrons. However, it is known that hydrogen strongly modifies the neutron energy spectrum, which in turn affects the gamma-ray peaks amplitudes. The link between hydrogen fraction and gamma ray peaks amplitude being complex, no attempt has been performed to inverse this link until now. Simulations show however that a neural network that takes as inputs the heights of carbon, oxygen and nitrogen gamma ray peaks can indeed determine the hydrogen fraction. Simulations of realistic cases show that the use of a neural network indeed allows identifying compounds having similar fractions of carbon, oxygen and nitrogen but different hydrogen fraction, opening the way to more accurate materials identification.

\section{Introduction}

$X$-ray systems are frequently used to identify unexpected items inside cargo containers, in order to search for smuggled goods or NRBC-E threats. However, since X-ray pictures do not allow identifying the chemical nature of the identified suspicious areas, to clear or confirm doubts, it can be necessary to open and check manually the container, which is time and resources consuming. For the same reason, a homogeneous container filled with forbidden or non-declared goods can pass unnoticed through X-ray systems. 
Neutron inspection systems which have been initially developed to detect NRBC threats can bring information on the chemical nature of transported goods and therefore can avoid the unnecessary manual inspection of compliant containers [Buffler]. Among these systems, the Associated Particle Technique (APT) [TPA] provides a 3D map of the nuclei inside the inspected volume. To achieve this goal, the APT uses the $14 \mathrm{MeV}$ neutron from the reaction ${ }^{2} \mathrm{H}+{ }^{3} \mathrm{H} \rightarrow n+\alpha$ to induce gamma rays in the inspected object, in coincidence with the alpha particle. Since the alpha particle is emitted with a $107^{\circ}$ angle relative to the neutron direction, the detection of the alpha particle with a position sensitive detector allows determining the direction of the $14 \mathrm{MeV}$ neutron. The time of flight measurement between the alpha particle and the neutron-induced gamma ray allows determining the gamma-ray origin. The nuclei that interacted with the neutrons are identified by analyzing the measured gamma-ray energy spectrum. In particular, APT offers the capability to identify organics, which constitute a major part of cargos, by determining the contributions of carbon, oxygen and nitrogen in measured gamma-ray spectra, using appropriate corrections [CorrFact].

However, since information concerning the quantity of hydrogen is missing, ambiguous measurements are unavoidable. For example, given measurement uncertainties, APT cannot make the difference between acetate ammonium, a benign material, and TNT, since these two compounds have almost the same $\mathrm{C}, \mathrm{N}, \mathrm{O}$ relative composition. To remove such ambiguities, information concerning the amount of hydrogen is compulsory, but neutron capture on hydrogen, leading to the emission of a $2.23 \mathrm{MeV}$ gamma-ray, has negligible cross section with fast neutrons and thus hydrogen cannot a-priori be measured using the APT.

Still, the presence of hydrogen will strongly modify the neutron energy spectrum through elastic scattering. This in turn has an impact on the measured gamma-ray energy spectra, since the $\left(n, n^{\prime}\right)$, $(n, \alpha), \ldots$ reactions, that produce the gamma-rays that serve to identify nuclei, strongly depend on the neutron energy, as illustrated for nitrogen in Figure 1 for $14 \mathrm{MeV}$ and $6 \mathrm{MeV}$ neutrons. Thus, the relative pic heights in the measured gamma-ray spectra contain information on the amount of hydrogen, but, given the complexity of the inverse problem, obtaining this information seems improbable.

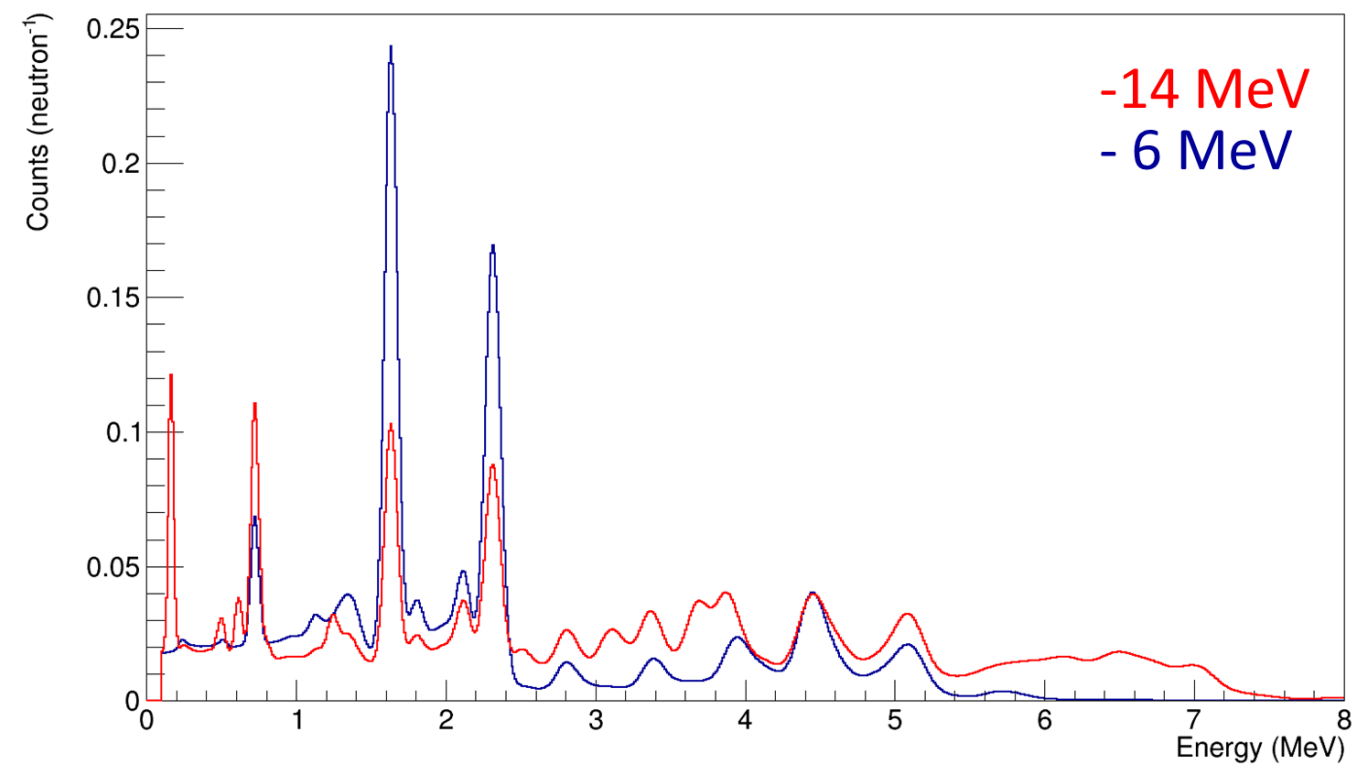




\section{Energy spectra processing with a neural network}

An attempt to determine the hydrogen fraction from the gamma-ray energy spectra shape has nevertheless been made by training a Neural Network (NN) with MCNPX simulations processed with our CERN's ROOT-based software able to reproduce realistic spectra taking into account the scintillation detectors energy and time resolution as well as counting statistics [MODAR].

The NN has been trained to provide $\mathrm{C}, \mathrm{N}, \mathrm{O}$ and $\mathrm{H}$ fractions, using as inputs the amplitudes, normalized by the tagged neutrons flux, of gamma-ray peaks induced by the fast neutrons on the carbon, oxygen and nitrogen of the organic material filling the container and on the iron of the container wall. A pre-selection of 11 peaks have been performed only keeping the gamma-ray peaks that can be isolated given the energy resolution of the $\mathrm{Nal}(\mathrm{TI})$ scintillation detectors. The energy of the pre-selected peaks are $6.13,5.106,4.439,3.684,2.742,2.313,2.125,1.632,1.238,0.847$, and $0.727 \mathrm{MeV}$. The training has been performed over 2500 training and 2500 control scenarios that are used to estimate the accuracy of the trained NN. The scenarios correspond to the setup presented in in Figure 2, which shows a system equipped with $205^{\prime \prime} \times 5^{\prime \prime} \times 10^{\prime \prime}$ Nal scintillation detectors placed in front of a cargo container. For each scenario, the container is filled with an organic matrix of random density and a random $\mathrm{C}, \mathrm{N}, \mathrm{O}$ and $\mathrm{H}$ composition. The $\mathrm{C}, \mathrm{N}, \mathrm{O}$ and $\mathrm{H}$ fractions, respectively $\mathrm{f}_{\mathrm{c}}, \mathrm{f}_{\mathrm{o}}, \mathrm{f}_{\mathrm{N}}$ and $f_{H}$, have been sampled uniformly in $[0 ; 1]$ with the conditions $f_{O, N, H} / f_{C}<10$ and $f_{H} / f_{O}<10$ to stay close to realistic chemical formulae of existing organic materials, whereas the density has been sampled uniformly in $[0 ; 1.5]$ thus covering almost all organic materials.

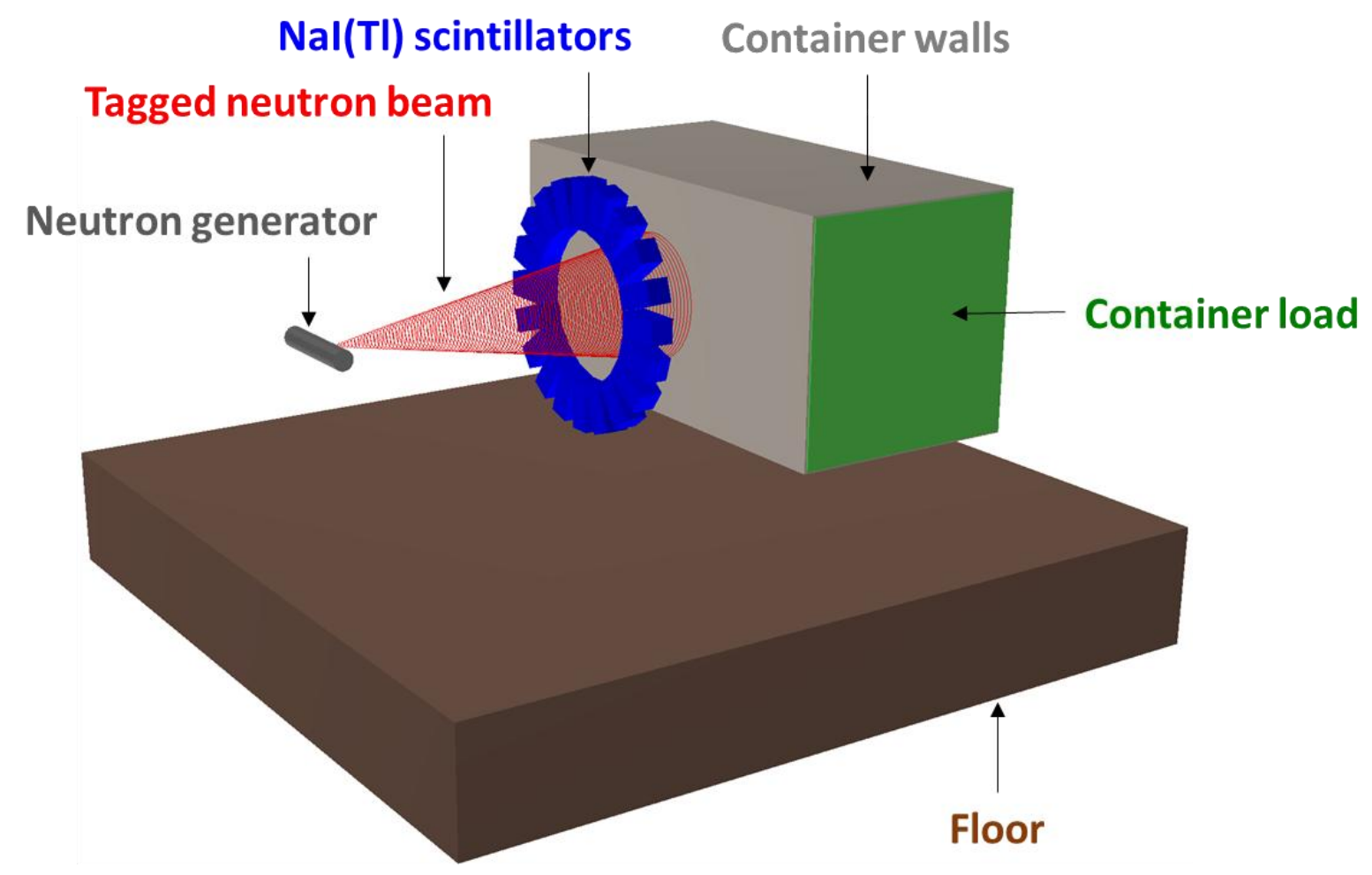

Figure 2 Illustration of the inspection of goods (green box) transported by a cargo container (grey box) with an APT system, showing the tagged neutron beam (red cone) emitted by a portable neutron generator (grey cylinder) and Nal scintillation detectors (bleu boxes) surrounding the neutron beam.

To reduce the number of entries, several $N N$ have been instantiated with input gamma ray sets omitting one of the reference gamma rays. For each of these $\mathrm{NN}$, the precision on the $\mathrm{C}, \mathrm{N}, \mathrm{O}$ and $\mathrm{H}$ fractions is compared to the ones obtained when keeping all gamma rays in the input set. Since the 
$\mathrm{NN}$ are trained stochastically, although they are trained with the same data set, two NN that process the same input vector will give slightly different results. Thus, to take into account the uncertainty related to the stochastic training, 100 batches made of 12 stochastically trained NN have been built. For each batch, a reference NN is trained with all of the 11 pre-selected gamma rays and 10 other NN are trained with one missing gamma ray, each of the $10 \mathrm{NN}$ being associated to a different missing gamma-ray peak.

For a given batch $i$, the $\mathrm{NN}$ using all gamma ray peaks excepted the one with energy $E$ determines the elemental fractions with an uncertainty (root mean square) $\sigma_{X, E}^{i}$, evaluated over the control set, $X$ standing for $\mathrm{C}, \mathrm{N}, \mathrm{O}$ or $\mathrm{H}$. On the other end, the reference $\mathrm{NN}$ from batch $i$ that keeps all gamma-rays provides the elemental fractions with a reference uncertainty $\sigma_{X, 0}^{i}$. With the 100 batches of $\mathrm{NN}$, one can therefore calculate an average sensitivity $s_{X, E}$ of the element $X$ to the gamma ray with energy $E$ as $S_{X, E}=\frac{1}{100} \sum_{i=1}^{100} \frac{\sigma_{X, E}^{i}-\sigma_{X, 0}^{i}}{\sigma_{X, 0}^{i}}$, with an uncertainty $\sigma_{S_{X, E}}=\sqrt{\frac{1}{100} \sum_{i=1}^{100}\left(\left(\frac{\sigma_{X, E}^{i}-\sigma_{X, 0}^{i}}{\sigma_{X, 0}^{i}}\right)-s_{X, E}\right)^{2}}$. A positive $s_{X, E}$ means that if the gamma ray with energy $E$ is removed from the training set, the precision on the fraction of element $X$ is deteriorated. A negative $s_{X, E}$ would indicate that if the gamma ray with energy $E$ is missing, the precision on the fraction of element $X$ is improved. The case $s_{X, E}=0$ means that the gamma ray with energy $E$ has no impact on the fraction of element $X$.

The sensitivities $s_{X, E}$ and their uncertainties shown in Figure 3 indicate that only the gamma-rays with energy $6.13,4.439,3.684$ and $0.847 \mathrm{MeV}$ seem significant for determining the elemental fractions. These gamma rays correspond to the reaction channels with the highest cross sections for Fe $(785 \mathrm{mb}), \mathrm{O}(148 \mathrm{mb}), \mathrm{C}(187 \mathrm{mb})$ and $\mathrm{N}(30 \mathrm{mb})$. It is remarkable that the nitrogen fraction is mostly sensitive to gamma rays coming from reactions that do not involve nitrogen. Since the gamma ray production cross sections on nitrogen are small compared to carbon, oxygen and iron, the nitrogen fraction seems better determined using its influence on the gamma ray spectra associated to the 3 other elements.

Keeping only these four gamma rays leads to a $\mathrm{NN}$ that is able to determine the $\mathrm{C}, \mathrm{N}, \mathrm{O}$ and $\mathrm{H}$ fractions with a relative uncertainty evaluated on the control set of $0.037,0.072,0.041$ and 0.027 , respectively. Keeping all gamma rays improves the precision on $\mathrm{C}, \mathrm{N}, \mathrm{O}$, and $\mathrm{H}$ fractions by $11 \%, 33 \%$, $39 \%$ and $11 \%$ respectively. Thus, it could be interesting to keep more gamma rays. However, since the weight of the containers is measured, one can add the freight density in the NN input vector. Doing so improves by about a factor 2 the accuracy of the $\mathrm{NN}$ trained with the four gamma rays, since it leads to $\mathrm{C}, \mathrm{N}, \mathrm{O}$ and $\mathrm{H}$ respective uncertainties equal to $0.025,0.033,0.019$ and 0.021 , while keeping all gamma rays leads to respective uncertainties equal to $0.025,0.029,0.019$ and 0.019 , which does not represent a significant improvement. Reducing the number of gamma ray peaks deteriorates significantly the precision of the NN. For example, keeping only $6.13,4.439$ and 0.847 $\mathrm{MeV}$ deteriorates the precision on $\mathrm{H}$ and $\mathrm{N}$ by about $60 \%$, the impact on oxygen being $20 \%$, keeping unchanged the precision on $\mathrm{C}$. Consequently, the density and the four selected gamma rays are sufficient to determine the $\mathrm{C}, \mathrm{N}, \mathrm{O}$ and $\mathrm{H}$ fractions.

It is also interesting to compare the NN performances against arithmetical approaches such as a backward stepwise regression. Indeed, performing a linear backward stepwise regression of order 2 with the 11 gamma-ray peaks and the density on the 5000 simulations leads to $\mathrm{C}, \mathrm{N}, \mathrm{O}$, and $\mathrm{H}$ fraction residual standard deviation of $0.050,0.029,0.031$ and 0.055 , respectively, showing that the NN handles the problem non-linearity in a better way. 


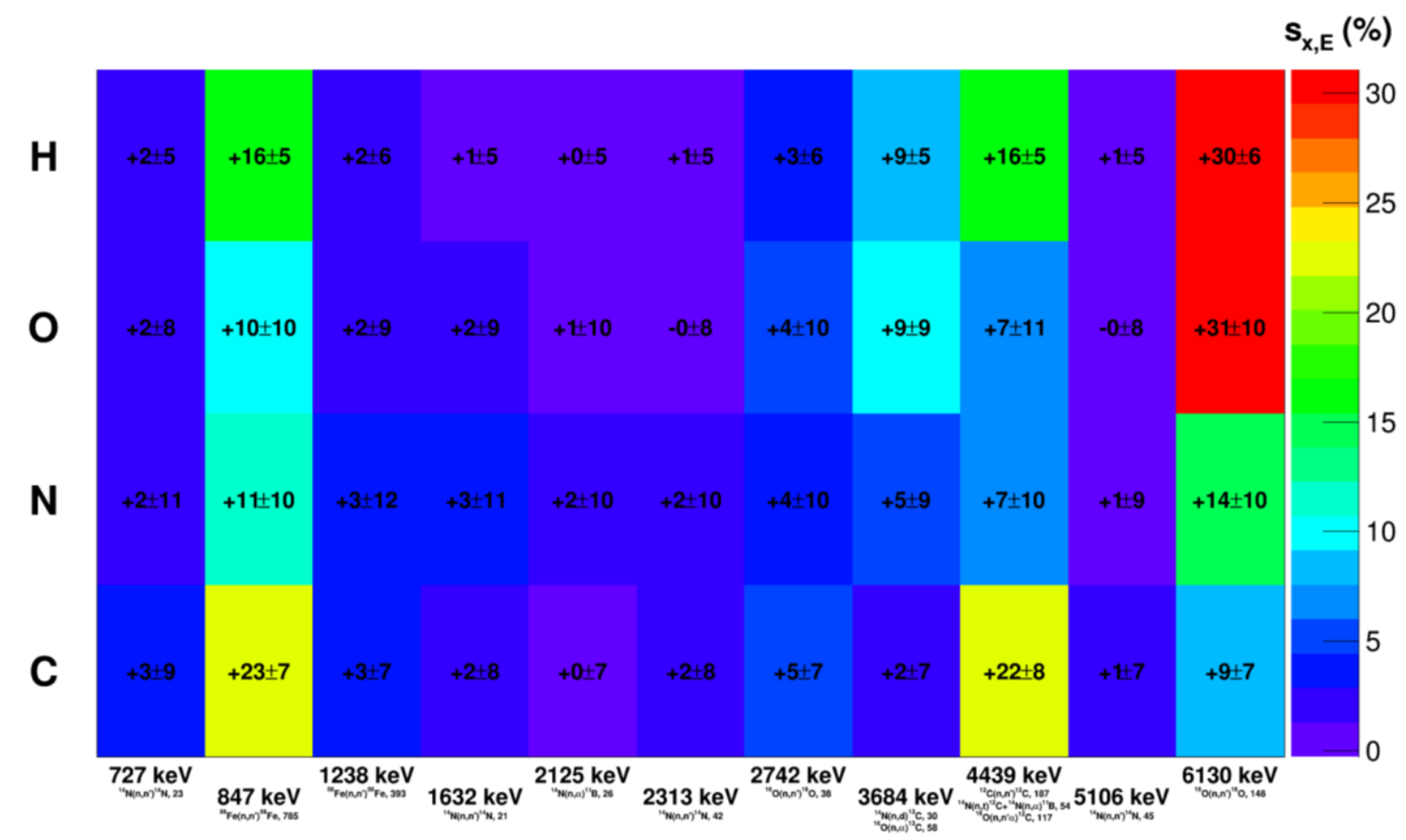

Figure 3 Sensitivity of the $C, N, O$, and $H$ fractions to the pre-selected gamma rays. The energy of each selected gamma ray shown in the $X$-axis is indicated with the associated reactions and the corresponding cross sections in mb for $14 \mathrm{MeV}$ neutrons obtained from [Simakov].

\section{Application on realistic scenarios}

The NN has been further tested on realistic simulated inspection scenarios that take into account statistical uncertainties corresponding to 10 minutes of measurements with a flux of $1 E 7$ tagged neutrons per second. The density of the container is a component of the input vector as well as the 4 selected gamma-rays. Examples of NN fraction determination with TNT, acetate ammonium and water are shown in Figure 4. The uncertainties take into account the NN precision stated above as well as statistical uncertainties. Uncertainties due to the limited counting statistics are estimated by Monte Carlo. The NN is applied on 100 versions the original energy histogram obtained from the MCNP calculation, each version histogram being obtained by sampling the original histogram bin content counts with a Poisson law. The statistical uncertainty on the $\mathrm{C}, \mathrm{N}, \mathrm{O}$ and $\mathrm{H}$ fraction is estimated using the root mean square calculated on the $100 \mathrm{C}, \mathrm{N}, \mathrm{O}$ and $\mathrm{H}$ fractions thus obtained with the duplicated energy spectra, but Figure 4 shows that the intrinsic $\mathrm{NN}$ uncertainty dominates. It is also interesting to note that within $2 \sigma$, the $\mathrm{C}, \mathrm{N}, \mathrm{O}$ and $\mathrm{H}$ fractions for water are correctly found although the carbon to oxygen ratio does not match the training condition $\mathrm{f}_{\mathrm{O}, \mathrm{N}, \mathrm{H}} / \mathrm{f}_{\mathrm{C}}<10$, thus showing the NN capability to extrapolate outside its training range. One can note that within $2 \sigma$, the backward stepwise regression is not always also able to find the correct elemental fractions. In particular, it cannot find the correct oxygen fraction in the case of water, showing the difficulty for such an approach to extrapolate outside its reference set. 


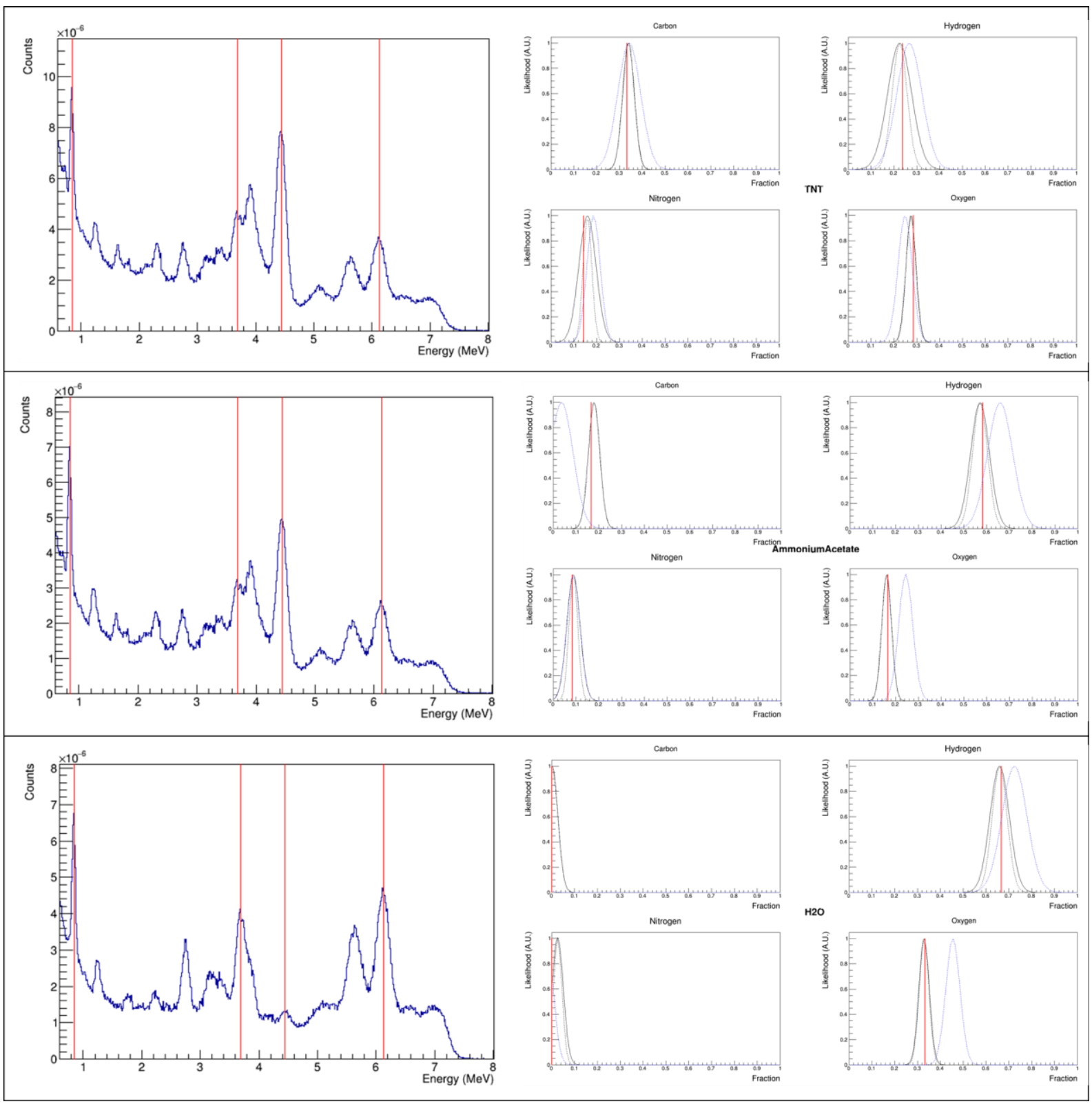

Figure $4 \mathrm{C}, \mathrm{N}, \mathrm{O}$ and $\mathrm{H}$ energy spectra (left) and fraction obtained with the NN (right) for TNT (top), acetate ammonium (middle) and water (bottom) corresponding to 10 minutes of measurements with a flux of $1 E 7$ tagged neutrons per second. The width of the likelihood functions takes into account the NN uncertainties obtained with the control cases, as well as the statistical uncertainties. The vertical lines show the position of the selected gamma ray peaks in the energy spectra and the true elemental composition in the fraction plots. The full lines black Gaussian on the right show the assumed Gaussian distribution of the elements fraction taking into account the NN resolution as well as the counting statistics, whereas the dashed lines only take into account the NN uncertainty determined after training with the control set. The dashed blue Gaussians show the backward stepwise regression expectation.

Such results are remarkable and open perspectives for more precise identification of organics, by replacing the $\mathrm{CNO}$ triangle diagram usually employed to identify organics with the $\mathrm{CNO}-\mathrm{H}$ diagram shown in Figure 6. For example, Figure 6 shows that it is now possible to differentiate between ammonium acetate and TNT, which is impossible to do with only carbon, oxygen and nitrogen fractions. Figure 6 also shows that using the hydrogen fraction, one is now able to better identify benign materials, thus allowing customs identifying wrongly declared goods. 


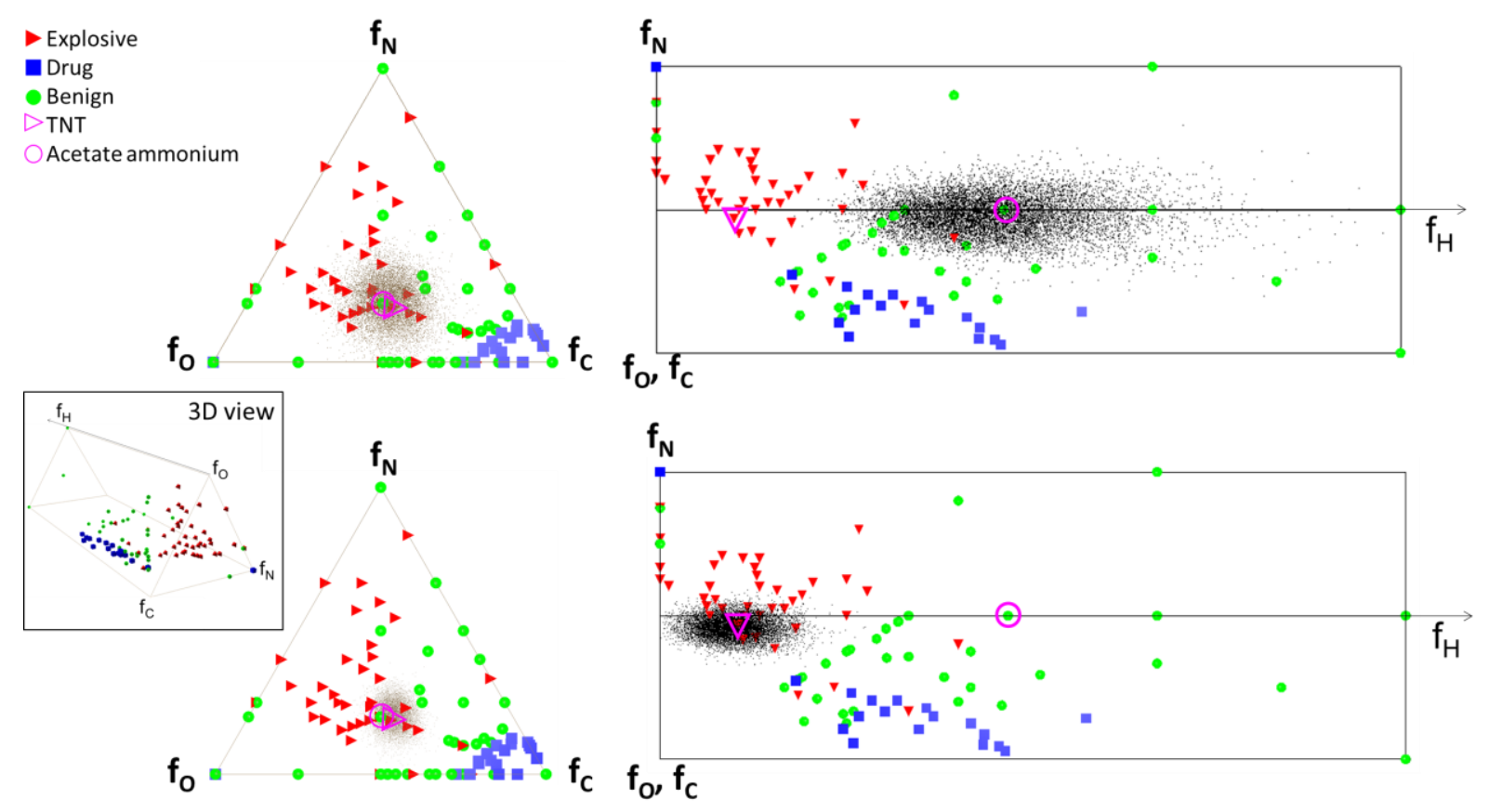

Figure 5 Simulated measurement of a cargo container loaded with acetate ammonium (top) and TNT (bottom), after 10 minutes irradiation with a tagged neutron flux $5 E 7 \mathrm{~s}^{-1}$. The black scattered points represent the NN values obtained for different energy spectra randomly sampled according to a Poisson law, to account for counting statistics. To ease the perception of the two dimensional projected CNO-H diagram (right), the enclosed box in the left shows the CNO two dimensional triangle diagram and the three dimensional view of $\mathrm{CNO}-\mathrm{H}$ diagram showing the $f_{H}$ axis. For a better understanding of the 2D plots, a 3D view of the triangular prism built with the fractions of carbon, oxygen, nitrogen and hydrogen is also shown.

\section{Conclusion}

Simulations show that fast neutron-induced gamma-ray energy spectra obtained using the Associated Particle Technique can be processed with a neural network to determine the fraction of hydrogen in organic compounds transported by cargo containers despite the absence of hydrogen gamma-ray peaks. The neural network input is a vector the components of which are the material density and peaks amplitudes of gamma rays induced by the fast neutrons on the carbon, oxygen and nitrogen of the organic compound and on the iron of the cargo container walls. The uncertainties obtained in 10-minute measurement $5 \mathrm{E} 7 \mathrm{n} / \mathrm{s}$ are sufficient to remove ambiguities between most benign organic materials and explosives, improving the search for contraband. These results will be followed by experiments aiming at further investigating the capability of this new approach to identify organics based on their $\mathrm{C}, \mathrm{N}, \mathrm{O}$ and $\mathrm{H}$ fractions.

[Buffler] A. Buffler, J. Tickner, Detecting contraband using neutrons: Challenges and future directions, Radiation Measurements 45 (2010) 1186-1192.

[TPA] B. Pérot, C. Carasco, A. Mariani, J.-L. Ma, S. Dejoies, W. El Kanawati, C. Eleon, Applications of the Associated Particle Technique, 2009 1st International Conference on Advancements in Nuclear Instrumentation, Measurement Methods and their Applications.

[CorrFactor] El Kanawati, B. Perot, C. Carasco, C. Eléon, V. Valkovic, D. Sudac, J. Obhodas, Conversion Factors from Counts to Chemical Ratios for the EURITRACK Tagged Neutron Inspection System, Nucl. Instr. and Meth in Physics Research A 654, 621-629 (2011). 
[MODAR] C.Carasco, MCNP output data analysis with ROOT (MODAR), Computer Physics Communications, Volume 181, Issue 12, December 2010, Pages 2210-2211.

[Simakov] S. P. Simakov, A. Pavlik, H. Vonach, and S. Hlavac, Status of experimental and evaluated discrete gamma-ray production at En=14.5 MeV Available from http, IAEA Nuclear Data Section, INDC(CCP)-413, 1998. 